\title{
Stability and Passivity of Spatially and Temporally Complex Dynamical Networks with Time-Varying Delays
}

\author{
Shun-Yan Ren ${ }^{1}$ and Yue-Hui Zhao ${ }^{2}$ \\ ${ }^{1}$ School of Computer Science \& Software Engineering, Tianjin Polytechnic University, Tianjin 300387, China \\ ${ }^{2}$ College of Post and Telecommunication of WIT, Wuhan 430070, China
}

Correspondence should be addressed to Shun-Yan Ren; renshunyan@163.com

Received 7 May 2014; Accepted 16 August 2014; Published 3 September 2014

Academic Editor: Zhichun Yang

Copyright (C) 2014 S.-Y. Ren and Y.-H. Zhao. This is an open access article distributed under the Creative Commons Attribution License, which permits unrestricted use, distribution, and reproduction in any medium, provided the original work is properly cited.

This paper proposes a new complex dynamical network model, in which the state, input, and output variables are varied with the time and space variables. By utilizing the Lyapunov functional method combined with the inequality techniques, several criteria for passivity and global exponential stability are established. Finally, numerical simulations are given to illustrate the effectiveness of the obtained results.

\section{Introduction}

Complex networks can be seen everywhere, which have become an important part of our daily life. Recently, the topology and dynamical behavior of complex dynamical networks have been extensively studied by the researchers. In particular, special attention has been focused on synchronization in complex dynamical networks, and many interesting results on synchronization were derived for various complex networks [1-10].

To our knowledge, in most existing works on the complex networks, they always assume that the node state is only dependent on the time. However, such simplification does not match the peculiarities of some real networks. Food webs are among the most well-known examples of complex networks and hold a central place in ecology to study the dynamics of animal populations. A food web can be characterized by a model of complex network, in which a node represents a species. To our knowledge, species are usually inhomogeneously distributed in a bounded habitat and the different population densities of predators and preys may cause different population movements; thus it is important and interesting to investigate their spatial density in order to better protect and control their population. In such a case, the state variable of node will represent the spatial density of the species. Moreover, the input and output variables are also dependent on the time and space in many practical situations. Therefore, it is essential to study the complex networks, in which the state, input, and output variables are varied with the time and space variables.

Recently, food web [11-24] has become a focal research topic and attracted increasing attention from many fields of scientific research. In [17], Pao discussed the asymptotic behavior of time-dependent solutions of a three-species reaction-diffusion system in a bounded domain under a Neumann boundary condition. Kim and Lin [21] considered the blowup properties of solutions for a parabolic system with homogeneous Dirichlet boundary conditions, which describes dynamic properties of three interacting species in a spatial habitat. As a natural extension of the existing network models, we propose a complex dynamical network with timevarying delays, which is described by a system of parabolic partial differential equations. In addition, we investigate the global exponential stability of the proposed network model.

Stability problems are often linked to the theory of dissipative systems, which postulate that the energy dissipated inside a dynamic system is less than that supplied from external source. Passivity is part of a broader and a general 
theory of dissipativeness. The main point of passivity theory is that the passive properties of systems can keep the systems internally stable. The passivity theory has found successful applications in diverse areas such as complexity [25], signal processing [26], stability $[27,28]$, chaos control and synchronization [29, 30], and fuzzy control [31]. Although research on passivity has attracted so much attention, little of that had been devoted to the passivity properties of spatially and temporally complex dynamical networks until Wang et al. [32] obtained some sufficient conditions on passivity properties for a class of reaction-diffusion neural networks with Dirichlet boundary conditions. To the best of our knowledge, very few researchers have investigated the passivity of complex dynamical networks with spatially and temporally varying state variables. Therefore, we also study the passivity of the proposed network model, and some sufficient conditions ensuring input strict passivity and output strict passivity are obtained by constructing appropriate Lyapunov functionals and using inequality techniques.

\section{Network Model and Preliminaries}

In this paper, we consider a parabolic complex network consisting of $N$ nonidentical nodes with diffusive and delay coupling. The entire network is described by

$$
\begin{gathered}
\frac{\partial w_{i}(x, t)}{\partial t}=d_{i} \Delta w_{i}(x, t)+p_{i} w_{i}(x, t)+u_{i}(x, t) \\
+\sum_{j=1}^{N} G_{i j} w_{j}\left(x, t-\tau_{j}(t)\right), \\
y_{i}(x, t)=g_{i} w_{i}(x, t)+h_{i} u_{i}(x, t),
\end{gathered}
$$

where $i=1,2, \ldots, N, \Omega=\left\{x=\left(x_{1}, x_{2}, \ldots, x_{m}\right)^{T}|| x_{k} \mid<\right.$ $\left.l_{k}, k=1,2, \ldots, m\right\}$ is the bounded domain with smooth boundary $\partial \Omega, d_{i} \in \mathbb{R}>0, p_{i}, g_{i}, h_{i} \in \mathbb{R},(x, t) \in \Omega \times \mathbb{R}^{+}, \tau_{j}(t)$ is the time-varying delay with $0 \leqslant \tau_{j}(t) \leqslant \tau, w_{i}(x, t) \in \mathbb{R}$ is the state of node $i, u_{i}(x, t), y_{i}(x, t)$ are the input and output of node $i$ at time $t$ and in space $x$, respectively, $\Delta=\sum_{k=1}^{m}\left(\partial^{2} / \partial x_{k}^{2}\right)$ is the Laplace diffusion operator on $\Omega$, and $G=\left(G_{i j}\right)_{N \times N}$ represents the topological structure of network and coupling strength between nodes, where $G_{i j}$ is defined as follows: if there is a connection from node $i$ to node $j(i \neq j)$, then $G_{i j}>0$; otherwise, $G_{i j}=0(i \neq j)$, and $G_{i i}=-\sum_{j=1, j \neq i}^{N} G_{i j}$

Let $w(x, t)=\left(w_{1}(x, t), w_{2}(x, t), \ldots, w_{N}(x, t)\right)^{T}$. The initial value and boundary value conditions associated with the network (1) are given in the form

$$
\begin{gathered}
w(x, t)=\Phi(x, t), \quad(x, t) \in \Omega \times[-\tau, 0], \\
\Phi(x, t)=\left(\phi_{1}(x, t), \phi_{2}(x, t), \ldots, \phi_{N}(x, t)\right)^{T}, \\
w(x, t)=0, \quad(x, t) \in \partial \Omega \times[-\tau,+\infty),
\end{gathered}
$$

where $\phi_{i}(x, t)(i=1,2, \ldots, N)$ is bounded and continuous on $\Omega \times[-\tau, 0]$. Let $w(x, t, \Phi)$ be the state trajectory of network (1) from the above initial value and boundary value conditions.
Next, we introduce some notations and definitions.

For any $e(x, t)=\left(e_{1}(x, t), e_{2}(x, t), \ldots, e_{N}(x, t)\right)^{T} \in \mathbb{R}^{N}$, $\|e(x, t)\|_{2}$ denotes

$$
\|e(x, t)\|_{2}=\left(\int_{\Omega} \sum_{i=1}^{N} e_{i}^{2}(x, t) d x\right)^{1 / 2}
$$

for any $\Theta(x, t)=\left(\theta_{1}(x, t), \theta_{2}(x, t), \ldots, \theta_{N}(x, t)\right)^{T} \in \mathbb{R}^{N}$, $(x, t) \in \Omega \times[-\tau, 0]$, we define

$$
\|\Theta\|_{\tau}=\sup _{-\tau \leqslant t \leqslant 0}\|\Theta(x, t)\|_{2}
$$

Definition 1. The complex network (1) is said to be globally exponentially stable if there exist constants $\epsilon>0$ and $M \geqslant$ 1 such that for any two solutions $w(x, t, \Phi), w(x, t, \Psi)$ of network (1) with initial functions $\Phi, \Psi$, respectively, it holds that

$$
\|w(x, t, \Psi)-w(x, t, \Phi)\|_{2} \leqslant M\|\Psi-\Phi\|_{\tau} e^{-\epsilon t}
$$

for all $t \geqslant 0$. If such $w(x, t, \Phi)$ is an equilibrium solution (or periodic solution), then this equilibrium solution (or periodic solution) is said to be globally exponentially stable.

Definition 2 (see [32]). A system with input $u(x, t)$ and output $y(x, t)$ where $u(x, t), y(x, t) \in \mathbb{R}^{p}$ is said to be passive if there is a constant $\beta \in \mathbb{R}$ such that

$$
\int_{0}^{t_{p}} \int_{\Omega} y^{T}(x, s) u(x, s) d x d s \geqslant-\beta^{2}
$$

for all $t_{p} \geqslant 0$, where $\Omega$ is a bounded compact set. If in addition, there are constants $\gamma_{1} \geqslant 0$ and $\gamma_{2} \geqslant 0$ such that

$$
\begin{gathered}
\int_{0}^{t_{p}} \int_{\Omega} y^{T}(x, s) u(x, s) d x d s \\
\geqslant-\beta^{2}+\gamma_{1} \int_{0}^{t_{p}} \int_{\Omega} u^{T}(x, s) u(x, s) d x d s \\
\quad+\gamma_{2} \int_{0}^{t_{p}} \int_{\Omega} y^{T}(x, s) y(x, s) d x d s
\end{gathered}
$$

for all $t_{p} \geqslant 0$, then the system is input-strictly passive if $\gamma_{1}>0$ and output-strictly passive if $\gamma_{2}>0$.

Remark 3. In [23], Wang and Wu discussed the global exponential stability and passivity of a parabolic complex network. In network model (1), the coupling matrix $G$ is diffusive. Namely, $G_{i i}=-\sum_{j=1, j \neq i}^{N} G_{i j}, i=1,2, \ldots, N$. Compared with the network model considered in [23], the network 
model considered in this paper may be more reasonable. On the other hand, we investigate the input strict passivity and output strict passivity of the complex network (1), which are totally different from the work in [23].

Lemma 4 (see [33]). Let $\Omega$ be a cube $\left|x_{k}\right|<l_{k}(k=$ $1,2, \ldots, m)$ and let $h(x)$ be a real-valued function belonging to $C^{1}(\Omega)$ which vanishes on the boundary $\partial \Omega$ of $\Omega$, that is, $\left.h(x)\right|_{\partial \Omega}=0$. Then,

$$
\int_{\Omega} h^{2}(x) d x \leqslant l_{k}^{2} \int_{\Omega}\left(\frac{\partial h}{\partial x_{k}}\right)^{2} d x
$$

where $x=\left(x_{1}, x_{2}, \ldots, x_{m}\right)^{T}$.

\section{Stability and Passivity of Parabolic Complex Network}

\subsection{Stability Analysis}

Theorem 5. Let $\dot{\tau}_{j}(t) \leqslant \sigma<1$. The complex network (1) is globally exponentially stable if there exist constants $\lambda_{i}>0$ and $\epsilon>0$ such that

$$
\begin{aligned}
2 \lambda_{i} \epsilon & +2 \lambda_{i} p_{i}-2 \lambda_{i} \sum_{k=1}^{m} \frac{d_{i}}{l_{k}^{2}} \\
& +\sum_{j=1}^{N} \frac{\lambda_{j}\left|G_{j i}\right| e^{2 \epsilon \tau}}{1-\sigma}+\lambda_{i} \sum_{j=1}^{N}\left|G_{i j}\right| \leqslant 0,
\end{aligned}
$$

where $i, j=1,2, \ldots, N$.

Proof. Firstly, we can get from (1) that

$$
\begin{aligned}
\frac{\partial w_{i}(x, t)}{\partial t}= & d_{i} \Delta w_{i}(x, t)+p_{i} w_{i}(x, t) \\
& +u_{i}(x, t)+\sum_{j=1}^{N} G_{i j} w_{j}\left(x, t-\tau_{j}(t)\right),
\end{aligned}
$$

where $i=1,2, \ldots, N$.

Define $z(x, t)=w(x, t, \Psi)-w(x, t, \Phi), \Psi_{z}(x, t)=$ $\Psi(x, t)-\Phi(x, t)$, and then the dynamics of the difference vector $z(x, t)=\left(z_{1}(x, t), z_{2}(x, t), \ldots, z_{N}(x, t)\right)^{T}$ is governed by the following equation:

$$
\begin{aligned}
\frac{\partial z_{i}(x, t)}{\partial t}= & d_{i} \Delta z_{i}(x, t) \\
& +\sum_{j=1}^{N} G_{i j} z_{j}\left(x, t-\tau_{j}(t)\right)+p_{i} z_{i}(x, t),
\end{aligned}
$$

where $i=1,2, \ldots, N$. Define the following Lyapunov functional for the system (11):

$$
\begin{aligned}
V(t)=\sum_{i=1}^{N} \lambda_{i} & \left\{e^{2 \epsilon t} \int_{\Omega} z_{i}^{2}(x, t) d x+\frac{e^{2 \epsilon \tau}}{1-\sigma} \sum_{j=1}^{N}\left|G_{i j}\right|\right. \\
& \left.\times \int_{t-\tau_{j}(t)}^{t} \int_{\Omega} z_{j}^{2}(x, s) e^{2 \epsilon s} d x d s\right\} .
\end{aligned}
$$

Calculating the time derivative of $V(t)$ along the trajectory of system (11), we can get

$\dot{V}(t)$

$$
=\sum_{i=1}^{N} \lambda_{i} e^{2 \epsilon t}\left\{2 \epsilon \int_{\Omega} z_{i}^{2}(x, t) d x+\int_{\Omega} 2 z_{i}(x, t) \frac{\partial z_{i}(x, t)}{\partial t} d x\right.
$$

$$
+\frac{e^{2 \epsilon \tau}}{1-\sigma} \sum_{j=1}^{N}\left|G_{i j}\right| \int_{\Omega} z_{j}^{2}(x, t) d x
$$

$$
-\frac{e^{2 \epsilon \tau}}{1-\sigma} \sum_{j=1}^{N}\left(1-\dot{\tau}_{j}(t)\right)\left|G_{i j}\right| e^{-2 \epsilon \tau_{j}(t)}
$$

$$
\left.\times \int_{\Omega} z_{j}^{2}\left(x, t-\tau_{j}(t)\right) d x\right\}
$$

$$
\leqslant \sum_{i=1}^{N} \lambda_{i} e^{2 \epsilon t}\left\{\left(2 \epsilon+2 p_{i}\right) \int_{\Omega} z_{i}^{2}(x, t) d x\right.
$$

$$
+2 d_{i} \int_{\Omega} z_{i}(x, t) \Delta z_{i}(x, t) d x
$$

$$
+2 \int_{\Omega} z_{i}(x, t) \sum_{j=1}^{N} G_{i j} z_{j}\left(x, t-\tau_{j}(t)\right) d x
$$$$
+\frac{e^{2 \epsilon \tau}}{1-\sigma} \sum_{j=1}^{N}\left|G_{i j}\right| \int_{\Omega} z_{j}^{2}(x, t) d x
$$

$$
\left.-\sum_{j=1}^{N}\left|G_{i j}\right| \int_{\Omega} z_{j}^{2}\left(x, t-\tau_{j}(t)\right) d x\right\}
$$

From Green's theorem and the boundary condition, we have

$$
\int_{\Omega} z_{i}(x, t) \Delta z_{i}(x, t) d x=-\sum_{k=1}^{m} \int_{\Omega}\left(\frac{\partial z_{i}(x, t)}{\partial x_{k}}\right)^{2} d x
$$

According to Lemma 4, we can obtain

$$
\sum_{k=1}^{m} \int_{\Omega}\left(\frac{\partial z_{i}(x, t)}{\partial x_{k}}\right)^{2} d x \geqslant \sum_{k=1}^{m} \int_{\Omega} \frac{z_{i}^{2}(x, t)}{l_{k}^{2}} d x .
$$


Therefore,

$\dot{V}(t)$

$$
\begin{aligned}
\leqslant \sum_{i=1}^{N}\left\{\lambda_{i}\left(2 \epsilon+2 p_{i}-2 \sum_{k=1}^{m} \frac{d_{i}}{l_{k}^{2}}\right) \int_{\Omega} z_{i}^{2}(x, t) d x\right. \\
+\frac{\lambda_{i} e^{2 \epsilon \tau}}{1-\sigma} \sum_{j=1}^{N}\left|G_{i j}\right| \int_{\Omega} z_{j}^{2}(x, t) d x \\
+2 \lambda_{i} \int_{\Omega} z_{i}(x, t) \sum_{j=1}^{N} G_{i j} z_{j}\left(x, t-\tau_{j}(t)\right) d x \\
\left.\quad-\lambda_{i} \sum_{j=1}^{N}\left|G_{i j}\right| \int_{\Omega} z_{j}^{2}\left(x, t-\tau_{j}(t)\right) d x\right\} e^{2 \epsilon t}
\end{aligned}
$$$$
\leqslant \sum_{i=1}^{N}\left\{\lambda_{i}\left(2 \epsilon+2 p_{i}-2 \sum_{k=1}^{m} \frac{d_{i}}{l_{k}^{2}}\right) \int_{\Omega} z_{i}^{2}(x, t) d x\right.
$$$$
+2 \lambda_{i} \sum_{j=1}^{N}\left|G_{i j}\right| \sqrt{\int_{\Omega} z_{i}^{2}(x, t) d x}
$$$$
\times \sqrt{\int_{\Omega} z_{j}^{2}\left(x, t-\tau_{j}(t)\right) d x}
$$$$
+\frac{\lambda_{i} e^{2 \epsilon \tau}}{1-\sigma} \sum_{j=1}^{N}\left|G_{i j}\right| \int_{\Omega} z_{j}^{2}(x, t) d x
$$$$
\left.-\lambda_{i} \sum_{j=1}^{N}\left|G_{i j}\right| \int_{\Omega} z_{j}^{2}\left(x, t-\tau_{j}(t)\right) d x\right\} e^{2 \epsilon t}
$$$$
\leqslant e^{2 \epsilon t} \sum_{i=1}^{N}\left\{\left(2 \lambda_{i} \epsilon+2 \lambda_{i} p_{i}-2 \lambda_{i} \sum_{k=1}^{m} \frac{d_{i}}{l_{k}^{2}}+\lambda_{i} \sum_{j=1}^{N}\left|G_{i j}\right|\right)\right.
$$$$
\times \int_{\Omega} z_{i}^{2}(x, t) d x+\frac{\lambda_{i} e^{2 \epsilon \tau}}{1-\sigma} \sum_{j=1}^{N}\left|G_{i j}\right|
$$$$
\left.\times \int_{\Omega} z_{j}^{2}(x, t) d x\right\}
$$$$
=e^{2 \epsilon t} \sum_{i=1}^{N}\left\{\left(2 \lambda_{i} \epsilon+2 \lambda_{i} p_{i}-2 \lambda_{i} \sum_{k=1}^{m} \frac{d_{i}}{l_{k}^{2}}+\lambda_{i} \sum_{j=1}^{N}\left|G_{i j}\right|\right.\right.
$$$$
\left.\left.+\sum_{j=1}^{N} \frac{\lambda_{j}\left|G_{j i}\right| e^{2 \epsilon \tau}}{1-\sigma}\right) \times \int_{\Omega} z_{i}^{2}(x, t) d x\right\} .
$$

From (9), we can get

$$
\dot{V}(t) \leqslant 0 \text {. }
$$

So $V(t) \leqslant V(0), t \geqslant 0$. Since

$$
\begin{gathered}
V(0)=\sum_{i=1}^{N} \lambda_{i}\left\{\int_{\Omega} z_{i}^{2}(x, 0) d x\right. \\
\left.+\frac{e^{2 \epsilon \tau}}{1-\sigma} \sum_{j=1}^{N}\left|G_{i j}\right| \int_{-\tau_{j}(0)}^{0} \int_{\Omega} z_{j}^{2}(x, s) e^{2 \epsilon s} d x d s\right\} \\
\leqslant\left[\max _{i=1,2, \ldots, N}\left\{\lambda_{i}\right\}+\max _{i=1,2, \ldots, N}\left\{\frac{\tau e^{2 \epsilon \tau}}{1-\sigma} \sum_{j=1}^{N} \lambda_{j}\left|G_{j i}\right|\right\}\right]\left\|\Psi_{z}\right\|_{\tau}^{2}, \\
V(t) \geqslant \min _{i=1,2, \ldots, N}\left\{\lambda_{i}\right\} e^{2 \epsilon t}\|z(x, t)\|_{2}^{2} .
\end{gathered}
$$

Let $\lambda_{-}=\min _{i=1,2, \ldots, N}\left\{\lambda_{i}\right\}, \lambda^{+}=\max _{i=1,2, \ldots, N}\left\{\lambda_{i}\right\}$, and

$$
M=\sqrt{\frac{\lambda^{+}+\max _{i=1,2, \ldots, N}\left\{\left(\tau e^{2 \epsilon \tau} /(1-\sigma)\right) \sum_{j=1}^{N} \lambda_{j}\left|G_{j i}\right|\right\}}{\lambda_{-}}} .
$$

Then $M \geqslant 1$, and we can obtain

$$
\|z(x, t)\|_{2} \leqslant M e^{-\epsilon t}\left\|\Psi_{z}\right\|_{\tau} .
$$

Namely,

$$
\|w(x, t, \Psi)-w(x, t, \Phi)\|_{2} \leqslant M\|\Psi-\Phi\|_{\tau} e^{-\epsilon t} .
$$

This completes the proof of Theorem 5 .

Practically, Theorem 5 not only can judge the global exponential stability of complex network (1), but also can guarantee the existence and uniqueness of the periodic solution in some circumstances.

Assume that $u_{i}(x, t)$ and $\tau_{i}(t)(i=1,2, \ldots, N)$ are periodic continuous functions with period $\omega$. Then we can get from (1) that

$$
\begin{aligned}
\frac{\partial w_{i}(x, t)}{\partial t}= & d_{i} \Delta w_{i}(x, t)+p_{i} w_{i}(x, t)+u_{i}(x, t) \\
& +\sum_{j=1}^{N} G_{i j} w_{j}\left(x, t-\tau_{j}(t)\right),
\end{aligned}
$$

where $u_{i}(x, t+\Phi)=u_{i}(x, t), \tau_{i}(t+\Phi)=\tau_{i}(t), i=1,2, \ldots, N$.

Theorem 6. Let $\dot{\tau}_{j}(t) \leqslant \sigma<1$. The system (22) has a unique $\omega$-periodic solution if there exist constants $\lambda_{i}>0$ and $\epsilon>0$ such that

$$
\begin{aligned}
2 \lambda_{i} \epsilon & +2 \lambda_{i} p_{i}-2 \lambda_{i} \sum_{k=1}^{m} \frac{d_{i}}{l_{k}^{2}} \\
& +\sum_{j=1}^{N} \frac{\lambda_{j}\left|G_{j i}\right| e^{2 \epsilon \tau}}{1-\sigma}+\lambda_{i} \sum_{j=1}^{N}\left|G_{i j}\right| \leqslant 0,
\end{aligned}
$$

where $i, j=1,2, \ldots, N$. 
Proof. Define

$$
\begin{aligned}
& w_{t}(\Psi)=w(x, t+s, \Psi), \quad s \in[-\tau, 0], t \geqslant 0 \\
& w_{t}(\Phi)=w(x, t+s, \Phi), \quad s \in[-\tau, 0], t \geqslant 0
\end{aligned}
$$

Obviously, from Theorem 5, we can derive

$$
\|w(x, t, \Psi)-w(x, t, \Phi)\|_{2} \leqslant M e^{-\epsilon t}\|\Psi-\Phi\|_{\tau}
$$

for $t \geqslant 0$, where $M \geqslant 1$. We can choose a positive integer $N$ such that

$$
M e^{-\epsilon(N \omega-\tau)} \leqslant \frac{1}{3}
$$

Now define a Poincaré mapping $P: C\left(\Omega \times[-\tau, 0], \mathbb{R}^{N}\right) \rightarrow$ $C\left(\Omega \times[-\tau, 0], \mathbb{R}^{N}\right)$ by

$$
\Psi \longrightarrow P(\Psi)=w_{\emptyset}(\Psi) .
$$

Since the periodicity of system (22), one has $P^{N}(\Psi)=$ $w_{N \omega}(\Psi)$. From (25) and (26), one obtains

$$
\left\|P^{N}(\Psi)-P^{N}(\Phi)\right\|_{\tau} \leqslant \frac{1}{3}\|\Psi-\Phi\|_{\tau}
$$

This implies that $P^{N}$ is a contraction mapping, so there exists one unique fixed point $\Psi^{*} \in C\left(\Omega \times[-\tau, 0], \mathbb{R}^{N}\right)$ such that

$$
P^{N}\left(\Psi^{*}\right)=\Psi^{*}
$$

Since $P^{N}\left(P\left(\Psi^{*}\right)\right)=P\left(P^{N}\left(\Psi^{*}\right)\right)=P\left(\Psi^{*}\right)$, so $P\left(\Psi^{*}\right)$ is also a fixed point of $P^{N}$, then

$$
P\left(\Psi^{*}\right)=\Psi^{*}
$$

Let $w\left(x, t, \Psi^{*}\right)$ be the solution of system (22) with initial conditions $\Psi^{*}$, then $w\left(x, t+\omega, \Psi^{*}\right)$ is also a solution of system (22). Obviously,

$$
\begin{array}{r}
w\left(x, t+\omega, \Psi^{*}\right)=w\left(x, t, w_{\omega}\left(x, \Psi^{*}\right)\right)=w\left(x, t, \Psi^{*}\right) \\
\forall t \geqslant 0 .
\end{array}
$$

This means that $w\left(x, t, \Psi^{*}\right)$ is exactly one $\omega$-periodic solution of system (22). This completes the proof of Theorem 6.

\subsection{Passivity Analysis}

Theorem 7. Let $\dot{\tau}_{j}(t) \leqslant \sigma<1$. The complex network (1) is input-strictly passive if there exist constants $\lambda_{i}>0$ and $\gamma>0$ such that

$$
\left(\begin{array}{cc}
M_{i} & \lambda_{i}-g_{i} \\
\lambda_{i}-g_{i} & \gamma-2 h_{i}
\end{array}\right) \leqslant 0,
$$

where $M_{i}=-\sum_{k=1}^{m}\left(2 \lambda_{i} d_{i} / l_{k}^{2}\right)+2 p_{i} \lambda_{i}+\lambda_{i} \sum_{j=1}^{N}\left|G_{i j}\right|+$ $\sum_{j=1}^{N}\left(\lambda_{j}\left|G_{j i}\right| /(1-\sigma)\right), i, j=1,2, \ldots, N$.
Proof. Firstly, construct a Lyapunov functional for the network (1) as follows:

$V(t)$

$$
\begin{aligned}
=\sum_{i=1}^{N} \lambda_{i}\left\{\int_{\Omega} w_{i}^{2}(x, t) d x\right. \\
\\
\left.\quad+\frac{1}{1-\sigma} \sum_{j=1}^{N}\left|G_{i j}\right| \int_{t-\tau_{j}(t)}^{t} \int_{\Omega} w_{j}^{2}(x, s) d x d s\right\} .
\end{aligned}
$$

Calculating the time derivative of $V(t)$ along the trajectory of system (1), we can get

$$
\begin{aligned}
\dot{V}(t)=\sum_{i=1}^{N} \lambda_{i}\left\{2 \int_{\Omega}\right. & w_{i}(x, t) \frac{\partial w_{i}(x, t)}{\partial t} d x \\
& +\frac{1}{1-\sigma} \sum_{j=1}^{N}\left|G_{i j}\right| \int_{\Omega} w_{j}^{2}(x, t) d x \\
& -\frac{1}{1-\sigma} \sum_{j=1}^{N}\left|G_{i j}\right|\left(1-\dot{\tau}_{j}(t)\right) \\
& \left.\times \int_{\Omega} w_{j}^{2}\left(x, t-\tau_{j}(t)\right) d x\right\}
\end{aligned}
$$$$
\leqslant \sum_{i=1}^{N} \lambda_{i}\left\{2 d_{i} \int_{\Omega} w_{i}(x, t) \Delta w_{i}(x, t) d x\right.
$$$$
+2 p_{i} \int_{\Omega} w_{i}^{2}(x, t) d x+2 \int_{\Omega} w_{i}(x, t) u_{i}(x, t) d x
$$$$
+2 \int_{\Omega} w_{i}(x, t) \sum_{j=1}^{N} G_{i j} w_{j}\left(x, t-\tau_{j}(t)\right) d x
$$$$
+\frac{1}{1-\sigma} \sum_{j=1}^{N}\left|G_{i j}\right| \int_{\Omega} w_{j}^{2}(x, t) d x
$$$$
\left.-\sum_{j=1}^{N}\left|G_{i j}\right| \int_{\Omega} w_{j}^{2}\left(x, t-\tau_{j}(t)\right) d x\right\}
$$$$
\leqslant \sum_{i=1}^{N} \lambda_{i}\left\{2 d_{i} \int_{\Omega} w_{i}(x, t) \Delta w_{i}(x, t) d x\right.
$$$$
+2 p_{i} \int_{\Omega} w_{i}^{2}(x, t) d x+2 \int_{\Omega} w_{i}(x, t) u_{i}(x, t) d x
$$$$
+\frac{1}{1-\sigma} \sum_{j=1}^{N}\left|G_{i j}\right| \int_{\Omega} w_{j}^{2}(x, t) d x
$$$$
+2 \sum_{j=1}^{N}\left|G_{i j}\right| \sqrt{\int_{\Omega} w_{i}^{2}(x, t) d x}
$$$$
\times \sqrt{\int_{\Omega} w_{j}^{2}\left(x, t-\tau_{j}(t)\right) d x}
$$$$
\left.-\sum_{j=1}^{N}\left|G_{i j}\right| \int_{\Omega} w_{j}^{2}\left(x, t-\tau_{j}(t)\right) d x\right\}
$$ 


$$
\begin{aligned}
\leqslant \sum_{i=1}^{N} \lambda_{i}\{2 & d_{i} \int_{\Omega} w_{i}(x, t) \Delta w_{i}(x, t) d x \\
& +2 p_{i} \int_{\Omega} w_{i}^{2}(x, t) d x+2 \int_{\Omega} w_{i}(x, t) u_{i}(x, t) d x \\
& +\frac{1}{1-\sigma} \sum_{j=1}^{N}\left|G_{i j}\right| \int_{\Omega} w_{j}^{2}(x, t) d x \\
& \left.+\sum_{j=1}^{N}\left|G_{i j}\right| \int_{\Omega} w_{i}^{2}(x, t) d x\right\}
\end{aligned}
$$

From Green's theorem and the boundary condition, we have

$$
\int_{\Omega} w_{i}(x, t) \Delta w_{i}(x, t) d x=-\sum_{k=1}^{m} \int_{\Omega}\left(\frac{\partial w_{i}(x, t)}{\partial x_{k}}\right)^{2} d x
$$

According to Lemma 4, we can obtain

$$
\sum_{k=1}^{m} \int_{\Omega}\left(\frac{\partial w_{i}(x, t)}{\partial x_{k}}\right)^{2} d x \geqslant \sum_{k=1}^{m} \int_{\Omega} \frac{w_{i}^{2}(x, t)}{l_{k}^{2}} d x .
$$

Therefore,

$$
\begin{aligned}
& \dot{V}(t)-2 \sum_{i=1}^{N} \int_{\Omega} y_{i}(x, t) u_{i}(x, t) d x+\gamma \sum_{i=1}^{N} \int_{\Omega} u_{i}^{2}(x, t) d x \\
& \leqslant \sum_{i=1}^{N}\left\{\left(-2 \lambda_{i} \sum_{k=1}^{m} \frac{d_{i}}{l_{k}^{2}}+\lambda_{i} \sum_{j=1}^{N}\left|G_{i j}\right|+2 p_{i} \lambda_{i}\right)\right. \\
& \times \int_{\Omega} w_{i}^{2}(x, t) d x+\left(\gamma-2 h_{i}\right) \int_{\Omega} u_{i}^{2}(x, t) d x \\
& +2\left(\lambda_{i}-g_{i}\right) \int_{\Omega} w_{i}(x, t) u_{i}(x, t) d x \\
& \left.+\frac{\lambda_{i}}{1-\sigma} \sum_{j=1}^{N}\left|G_{i j}\right| \int_{\Omega} w_{j}^{2}(x, t) d x\right\} \\
& =\sum_{i=1}^{N}\left\{\left(-2 \lambda_{i} \sum_{k=1}^{m} \frac{d_{i}}{l_{k}^{2}}+2 p_{i} \lambda_{i}\right.\right. \\
& \left.+\lambda_{i} \sum_{j=1}^{N}\left|G_{i j}\right|+\sum_{j=1}^{N} \frac{\lambda_{j}\left|G_{j i}\right|}{1-\sigma}\right) \int_{\Omega} w_{i}^{2}(x, t) d x \\
& +2\left(\lambda_{i}-g_{i}\right) \int_{\Omega} w_{i}(x, t) u_{i}(x, t) d x \\
& \left.+\left(\gamma-2 h_{i}\right) \int_{\Omega} u_{i}^{2}(x, t) d x\right\} \\
& =\sum_{i=1}^{N} \int_{\Omega}\left(\begin{array}{l}
w_{i}(x, t) \\
u_{i}(x, t)
\end{array}\right)^{T}\left(\begin{array}{cc}
M_{i} & \lambda_{i}-g_{i} \\
\lambda_{i}-g_{i} & \gamma-2 h_{i}
\end{array}\right)\left(\begin{array}{l}
w_{i}(x, t) \\
u_{i}(x, t)
\end{array}\right) d x .
\end{aligned}
$$

It follows from (32) that

$$
\begin{gathered}
\dot{V}(t)-2 \sum_{i=1}^{N} \int_{\Omega} y_{i}(x, t) u_{i}(x, t) d x \\
+\gamma \sum_{i=1}^{N} \int_{\Omega} u_{i}^{2}(x, t) d x \leqslant 0 .
\end{gathered}
$$

By integrating (38) with respect to $t$ over the time period 0 to $t_{p}$, we can get

$$
\begin{aligned}
& 2 \sum_{i=1}^{N} \int_{0}^{t_{p}} \int_{\Omega} y_{i}(x, t) u_{i}(x, t) d x d t \\
& \quad \geqslant V\left(t_{p}\right)-V(0)+\gamma \sum_{i=1}^{N} \int_{0}^{t_{p}} \int_{\Omega} u_{i}^{2}(x, t) d x d t .
\end{aligned}
$$

From the definition of $V(t)$, we have $V\left(t_{p}\right) \geqslant 0$ and $V(0) \geqslant 0$. Thus,

$$
\begin{aligned}
& \sum_{i=1}^{N} \int_{0}^{t_{p}} \int_{\Omega} y_{i}(x, t) u_{i}(x, t) d x d t \\
& \quad \geqslant-\beta^{2}+\frac{\gamma}{2} \sum_{i=1}^{N} \int_{0}^{t_{p}} \int_{\Omega} u_{i}^{2}(x, t) d x d t
\end{aligned}
$$

for all $t_{p} \geqslant 0$, where $\beta=\sqrt{V(0) / 2}$. The proof is completed.

By a minor modification of the proof of Theorem 7 , we can easily get the following.

Theorem 8. Let $\dot{\tau}_{j}(t) \leqslant \sigma<1$. The complex network (1) is output-strictly passive if there exist constants $\lambda_{i}>0$ and $\gamma>0$ such that

$$
\left(\begin{array}{cc}
\widehat{M}_{i} & \lambda_{i}-g_{i}+\gamma g_{i} h_{i} \\
\lambda_{i}-g_{i}+\gamma g_{i} h_{i} & \gamma h_{i}^{2}-2 h_{i}
\end{array}\right) \leqslant 0
$$

where $i, j=1,2, \ldots, N, \widehat{M}_{i}=-2 \lambda_{i} \sum_{k=1}^{m}\left(d_{i} / l_{k}^{2}\right)+2 p_{i} \lambda_{i}+$ $\lambda_{i} \sum_{j=1}^{N}\left|G_{i j}\right|+\gamma g_{i}^{2}+\sum_{j=1}^{N}\left(\lambda_{j}\left|G_{j i}\right| /(1-\sigma)\right)$.

Remark 9. In [22], two kinds of impulsive parabolic complex networks were considered, in which the node states are dependent on the time and space variables. Several global exponential stability and robust global exponential stability criteria were derived for the impulsive parabolic complex networks. Both global exponential stability and passivity are taken into consideration in this paper, and some sufficient conditions are established.

\section{Example}

In this section, an illustrative example is provided to verify the effectiveness of the proposed theoretical results. 


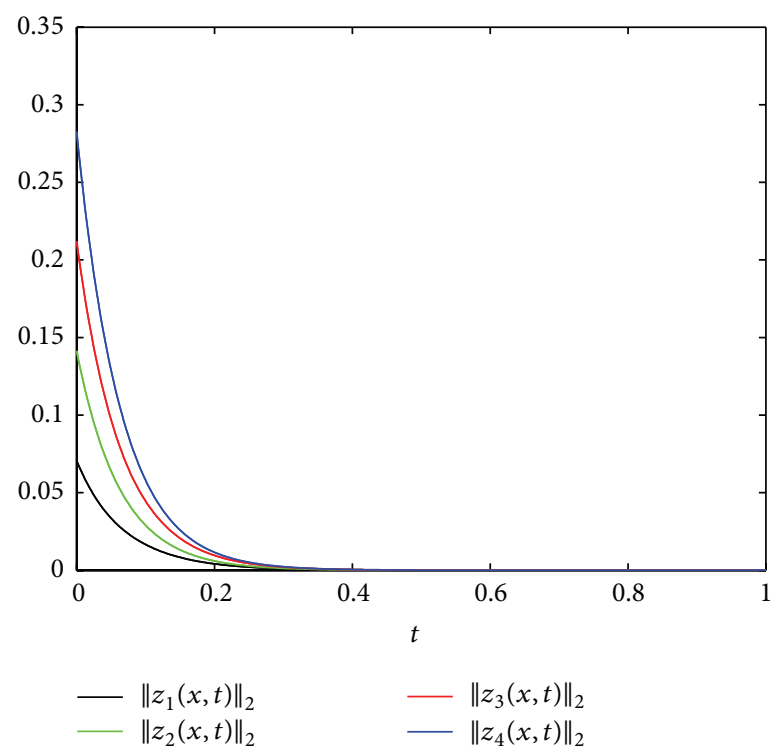

Figure 1: The change processes of $\left\|z_{i}(x, t)\right\|_{2}, i=1,2,3,4$.

Consider a complex network model, in which each node is a 1-dimensional dynamical system described by

$$
\begin{aligned}
& \frac{\partial w_{i}(x, t)}{\partial t} \\
& =d_{i} \frac{\partial^{2} w_{i}(x, t)}{\partial x^{2}}+p_{i} w_{i}(x, t), \quad i=1,2,3,4 .
\end{aligned}
$$

Take $d_{i}=0.4, p_{i}=0.1 i, g_{i}=1, h_{i}=2, \tau_{j}(t)=0.2-\left(e^{-t} /(4+\right.$ $j)), \tau=0.2, \sigma=0.2, x \in(-0.5,0.5)$. The matrix $G$ is chosen as

$$
\left(\begin{array}{cccc}
-0.3 & 0.1 & 0.15 & 0.05 \\
0.2 & -0.3 & 0.1 & 0 \\
0.05 & 0.05 & -0.3 & 0.2 \\
0.1 & 0 & 0.1 & -0.2
\end{array}\right)
$$

It is easy to verify that (9) is satisfied if $\lambda_{1}=\lambda_{2}=\lambda_{3}=$ $\lambda_{4}=1$ and $\epsilon=0.25$. From Theorem 5, complex network (1) with above given parameters is globally exponentially stable. Moreover, if we take $\lambda_{1}=\lambda_{2}=\lambda_{3}=\lambda_{4}=1$ and $\gamma=1.5$, then (32) is satisfied. According to Theorem 7, complex network (1) with above given parameters is also input-strictly passive. The simulation results are shown in Figures 1 and 2.

\section{Conclusion}

A parabolic complex network model has been introduced, in which the state, input, and output variables are dependent on the time and space variables. The input strict passivity, output strict passivity, and global exponential stability of the proposed network model have been discussed in this paper, and several sufficient conditions have been established. Illustrative simulations have been provided to verify the correctness and effectiveness of the obtained results. In future work, we shall study the passivity and robust passivity of parabolic complex networks with impulsive effects.

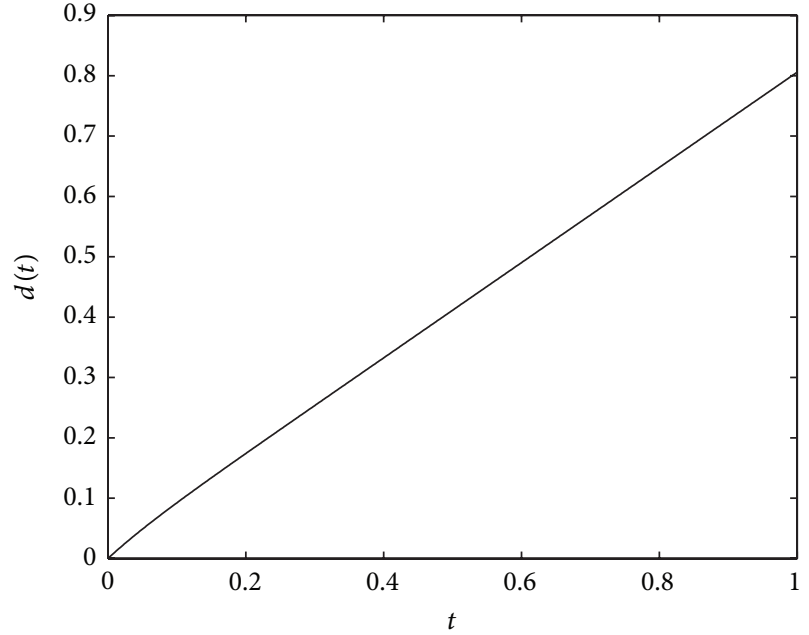

FIGURE 2: The change process of $d(t)=\sum_{i=1}^{4} \int_{0}^{t} \int_{\Omega} y_{i}(x, s) u_{i}$ $(x, s) d x d s-0.75 \sum_{i=1}^{4} \int_{0}^{t} \int_{\Omega} u_{i}^{2}(x, s) d x d s$, in which $u_{i}(x, t)=$ $0.2 i \sin (2 \pi x), i=1,2,3,4$.

\section{Conflict of Interests}

The authors declare that there is no conflict of interests regarding the publication of this paper.

\section{Acknowledgment}

The authors would like to thank Professor J.-L. Wang (Tianjin Polytechnic University, Tianjin, China) for his kind help.

\section{References}

[1] J. Lu and G. Chen, "A time-varying complex dynamical network model and its controlled synchronization criteria," IEEE Transactions on Automatic Control, vol. 50, no. 6, pp. 841-846, 2005.

[2] W. Yu, G. Chen, and J. Lü, "On pinning synchronization of complex dynamical networks," Automatica, vol. 45, no. 2, pp. 429-435, 2009.

[3] W. Yu, J. Cao, and J. Lu, "Global synchronization of linearly hybrid coupled networks with time-varying delay," SIAM Journal on Applied Dynamical Systems, vol. 7, no. 1, pp. 108-133, 2008.

[4] J. L. Wang, H. N. Wu, and L. Guo, "Novel adaptive strategies for synchronization of linearly coupled neural networks with reaction-diffusion terms," IEEE Transactions on Neural Networks and Learning Systems, vol. 25, pp. 429-440, 2014.

[5] J. L. Wang and H. N. Wu, "Synchronization and adaptive control of an array of linearly coupled reaction-diffusion neural networks with hybrid coupling," IEEE Transactions on Cybernetics, vol. 44, no. 8, pp. 1350-1361, 2014.

[6] J. L. Wang, Z. C. Yang, T. W. Huang, and M. Q. Xiao, "Synchronization criteria in complex dynamical networks with nonsymmetric coupling and multiple time-varying delays," Applicable Analysis, vol. 91, no. 5, pp. 923-935, 2012.

[7] J. L. Wang, Z. C. Yang, and T. W. Huang, "Local and global exponential synchronization of complex delayed dynamical networks with general topology," Discrete and Continuous Dynamical Systems B, vol. 16, no. 1, pp. 393-408, 2011. 
[8] J.-L. Wang and H.-N. Wu, "Local and global exponential output synchronization of complex delayed dynamical networks," Nonlinear Dynamics, vol. 67, no. 1, pp. 497-504, 2012.

[9] J. Wang and $\mathrm{H}$. Wu, "Synchronization criteria for impulsive complex dynamical networks with time-varying delay," Nonlinear Dynamics, vol. 70, no. 1, pp. 13-24, 2012.

[10] J. L. Wang and H. N. Wu, "Adaptive output synchronization of complex delayed dynamical networks with output coupling," Neurocomputing, vol. 142, pp. 174-181, 2014.

[11] M. D. Holland and A. Hastings, "Strong effect of dispersal network structure on ecological dynamics," Nature, vol. 456, no. 7223, pp. 792-795, 2008.

[12] J. M. Montoya, S. L. Pimm, and R. V. Solé, "Ecological networks and their fragility," Nature, vol. 442, no. 7100, pp. 259-264, 2006.

[13] W. Ko and I. Ahn, "Dynamics of a simple food chain model with a ratio-dependent functional response," Nonlinear Analysis: Real World Applications, vol. 12, no. 3, pp. 1670-1680, 2011.

[14] Y.-M. Wang, "Asymptotic behavior of solutions for a LotkaVolterra mutualism reaction-diffusion system with time delays," Computers \& Mathematics with Applications, vol. 58, no. 3, pp. 597-604, 2009.

[15] Y. M. Wang, "Asymptotic behavior of solutions for a class of predator-prey reaction-diffusion systems with time delays," Journal of Mathematical Analysis and Applications, vol. 328, no. 1, pp. 137-150, 2007.

[16] C. Wang, S. Wang, F. Yang, and L. Li, "Global asymptotic stability of positive equilibrium of three-species Lotka-Volterra mutualism models with diffusion and delay effects," Applied Mathematical Modelling, vol. 34, no. 12, pp. 4278-4288, 2010.

[17] C. V. Pao, "The global attractor of a competitor-competitormutualist reaction-diffusion system with time delays," Nonlinear Analysis: Theory, Methods and Applications, vol. 67, no. 9, pp. 2623-2631, 2007.

[18] C. V. Pao, "Global attractor of a coupled finite difference reaction diffusion system with delays," Journal of Mathematical Analysis and Applications, vol. 288, no. 1, pp. 251-273, 2003.

[19] C. V. Pao, "Convergence of solutions of reaction-diffusion systems with time delays," Nonlinear Analysis. Theory, Methods \& Applications, vol. 48, pp. 349-362, 2002.

[20] M. R. Garvie, "Finite-difference schemes for reaction-diffusion equations modeling predator-prey interactions in MATLAB," Bulletin of Mathematical Biology, vol. 69, no. 3, pp. 931-956, 2007.

[21] K. I. Kim and Z. Lin, "Blowup in a three-species cooperating model," Applied Mathematics Letters, vol. 17, no. 1, pp. 89-94, 2004.

[22] J. Wang and $\mathrm{H}$. Wu, "Stability analysis of impulsive parabolic complex networks," Chaos, Solitons and Fractals, vol. 44, no. 11, pp. 1020-1034, 2011.

[23] J. L. Wang and H. N. Wu, "Robust stability and robust passivity of parabolic complex networks with parametric uncertainties and time-varying delays," Neurocomputing, vol. 87, pp. 26-32, 2012.

[24] J.-L. Wang, H.-N. Wu, and L. Guo, "Pinning control of spatially and temporally complex dynamical networks with time-varying delays," Nonlinear Dynamics, vol. 70, no. 2, pp. 1657-1674, 2012.

[25] L. O. Chua, "Passivity and complexity", IEEE Transactions on Circuits and Systems. I. Fundamental Theory and Applications, vol. 46, no. 1, pp. 71-82, 1999.

[26] L. Xie, M. Fu, and H. Li, "Passivity analysis and passification for uncertain signal processing systems," IEEE Transactions on Signal Processing, vol. 46, no. 9, pp. 2394-2403, 1998.
[27] D. J. Hill and P. J. Moylan, "Stability results for nonlinear feedback systems," Automatica, vol. 13, no. 4, pp. 377-382, 1977.

[28] G. L. Santosuosso, "Passivity of nonlinear systems with inputoutput feedthrough," Automatica, vol. 33, no. 4, pp. 693-697, 1997.

[29] W. Yu, "Passive equivalence of chaos in Lorenz system," IEEE Transactions on Circuits and Systems I: Fundamental Theory and Applications, vol. 46, no. 7, pp. 876-878, 1999.

[30] C. W. Wu, "Synchronization in arrays of coupled nonlinear systems: passivity, circle criterion, and observer design," IEEE Transactions on Circuits and Systems. I. Fundamental Theory and Applications, vol. 48, no. 10, pp. 1257-1261, 2001.

[31] G. Calcev, R. Gorez, and M. De Neyer, "Passivity approach to fuzzy control systems," Automatica, vol. 34, no. 3, pp. 339-344, 1998.

[32] J. Wang, H. Wu, and L. Guo, "Passivity and stability analysis of reaction-diffusion neural networks with dirichlet boundary conditions," IEEE Transactions on Neural Networks, vol. 22, no. 12, pp. 2105-2116, 2011.

[33] J. G. Lu, "Global exponential stability and periodicity of reaction-diffusion delayed recurrent neural networks with Dirichlet boundary conditions," Chaos, Solitons and Fractals, vol. 35, no. 1, pp. 116-125, 2008. 


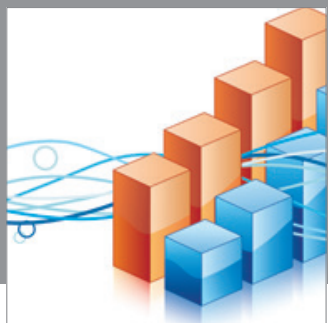

Advances in

Operations Research

mansans

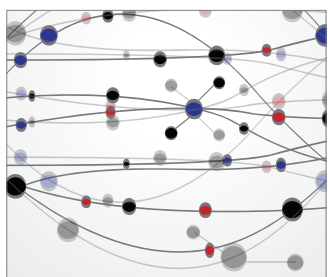

The Scientific World Journal
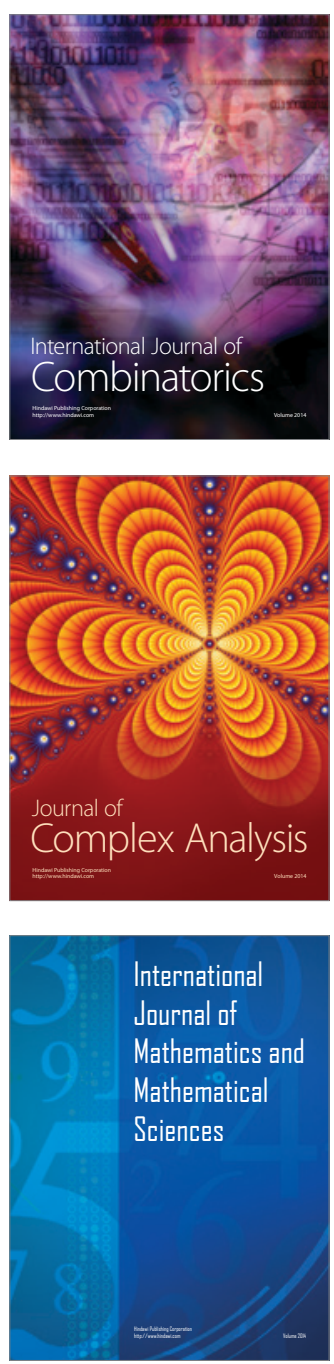
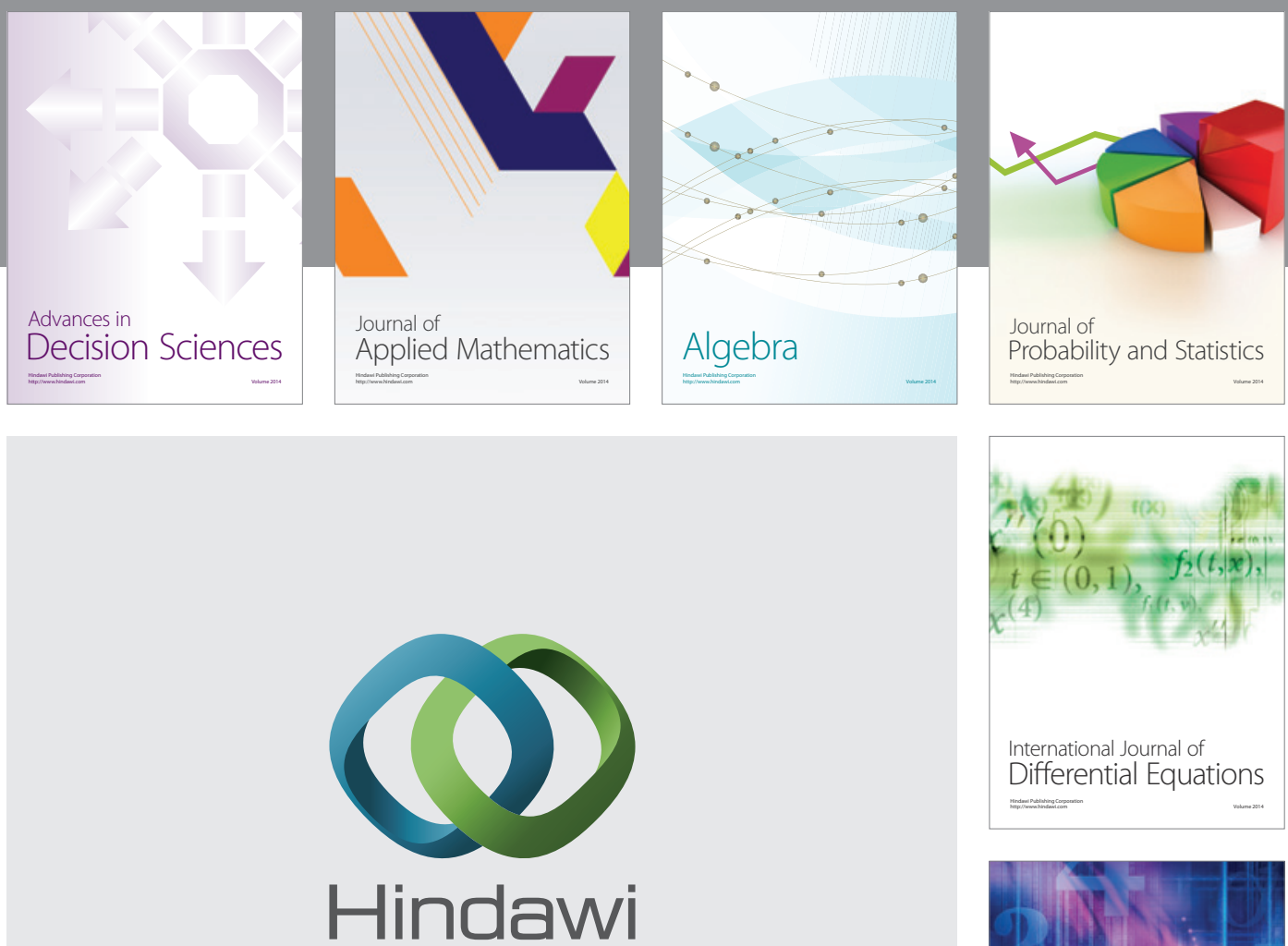

Submit your manuscripts at http://www.hindawi.com
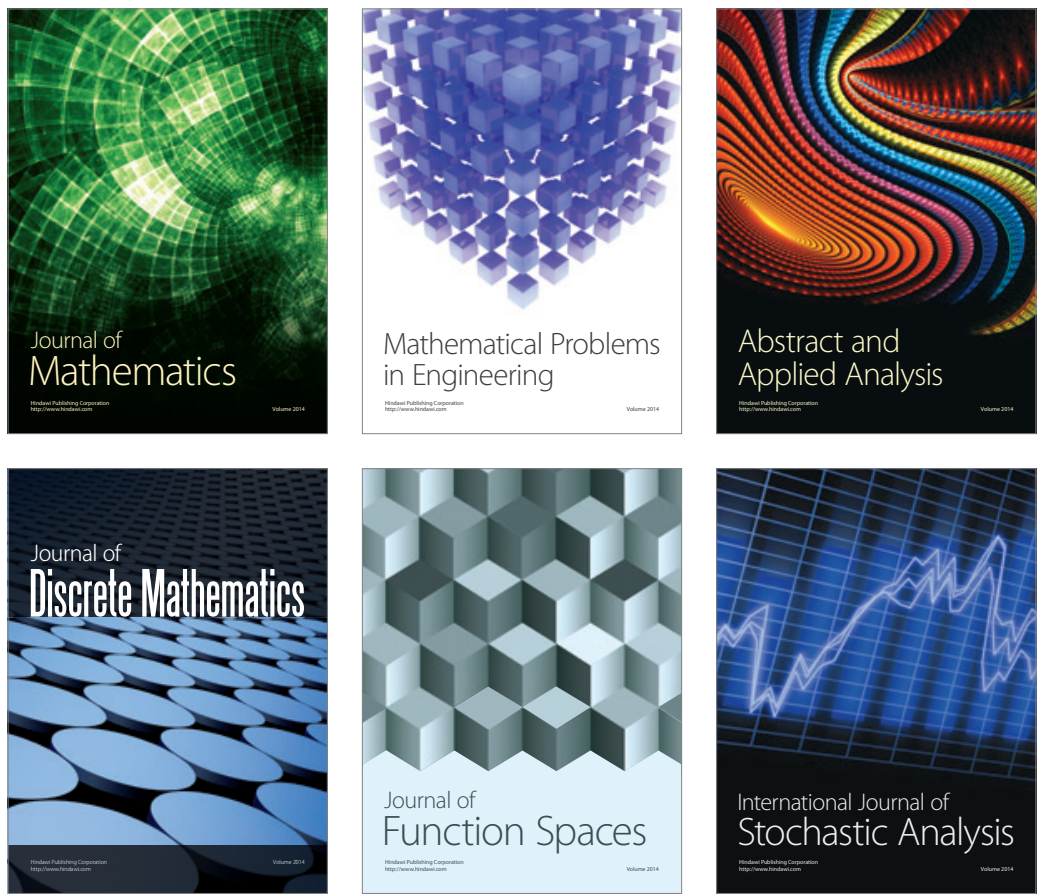

Journal of

Function Spaces

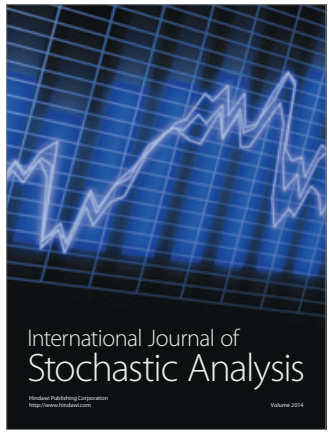

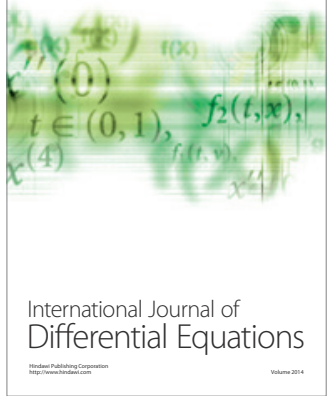
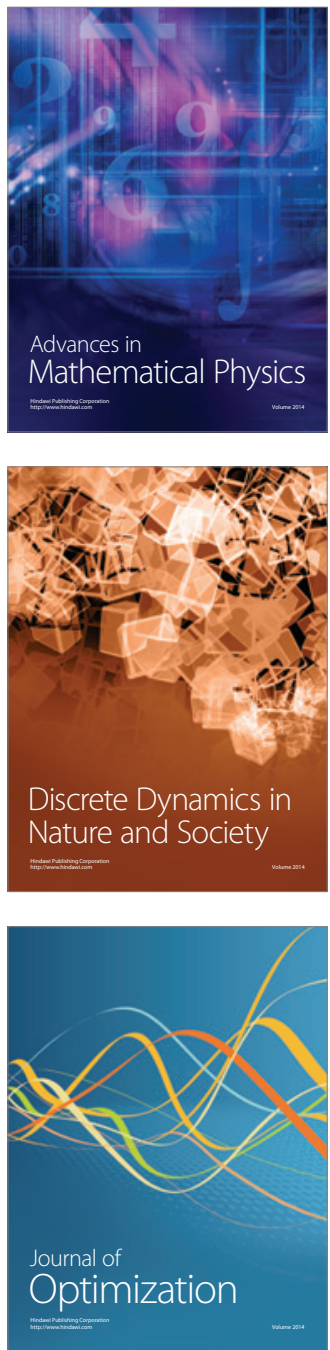